satisfaction of parents, referrers and children admitted to the residential child psychiatry unit at Guy's Hospital over a three year period.

Of the 50 children admitted to the unit, 25 carers, 22 children and 27 referrers responded. Of respondents $92 \%$ of carers, $82 \%$ of children and $85 \%$ of referrers found the admission helpful. Of the 13 responses that could be matched between carer, child and referrer, 12 agreed that admission was helpful.

Parents reported that the admission gave them greater understanding of the problem, increased children's confidence and gave parents a break while their child recovered. However, parents complained that other children were a bad influence on their child, the ward rules were too strict and some were concerned about insulation from the family.

Children reported that they now understood their problem, had more confidence, learnt to relax, could keep up their school work and made new friends. Children complained that they were homesick and unhappy, lacked privacy and that their particular problem was not solved.

Of referrers, $92 \%$ had received a summary. The intensive assessment had helped clarify problems and expert advice had helped parents cope, family functioning had improved and the children were more independent and confident. One referrer was unhappy as the problem still existed. Another felt that the mother's needs were not met.

As a consequence of these comments the unit has compiled a brochure and parents are involved more in the assessment and goal setting stages preceding admission.

KamaldeEP BHUI, SARAH DENNISON and TOM MORAN, Hector Cameron Ward, Guy's Hospital, London SE1 9RT

\section{Value of experience in child and family psychiatry}

Sir: I agree with Jan Hermsen's comments (Psychiatric Bulletin, 1993, 17, 628) on the value of experience in child and family psychiatry as part of general psychiatric training. It gives the trainee two specific advantages in treating adult patients: greater awareness of environmental and psychological influences on behaviour and emotion, and technical skills which make dealing with the families of adult patients less daunting and more therapeutically valuable. I often find it helpful to use my knowledge of systems theory, and techniques such as circular questioning, when faced by families attempting to cope with mental illness in an adult relative. It seems illogical to abandon a family approach when the patient reaches the age of 16 as childhood ex- periences and family environment have a crucial bearing on adult lives, including the development and experience of mental illness.

I was lucky enough to gain experience of child and family psychiatry at an early stage in my training, during my second senior house officer post. I found working as part of a multidisciplinary team that was determinedly (and appropriately) non-medical in outlook challenging but rewarding, and one that trainees are unlikely to encounter outside this field. Open discussion of emotions evoked by the nature of the work, for example as a result of over-identification with or projection of feelings onto children, fostered self-understanding. Personal psychotherapy was strongly encouraged as a further aid to self-knowledge and therapeutic effectiveness. (Indeed, I believe that local private psychotherapists made a reasonable living from seeing staff from the department!)

The benefits gained from working with children and families lead me to strongly support $\mathrm{Dr}$ Hermsen's suggestion that all trainees be encouraged to gain such experience but, in view of the shortage of posts, I fear that only a minority will be able to do so.

ADAM KIRBY, Windsor Clinic, Fazakerly Hospital, Liverpool L9 7AL

\section{Hypnosis and psychotherapy}

Sir: Interest in hypnosis has waxed and waned over the years. I have been on a course which guarantees to produce integral therapists. Almost anyone who pays the course fee, regardless of background, can subscribe to the course and at the end can declare him or herself a psychotherapist, adding a few initials after his or her name.

In a seminar given to senior registrars in child psychiatry I conveyed how I use hypnosis as a 'tool' for psychotherapy and my colleagues confirmed that hypnosis is a powerful medium to transmit psychotherapeutic interventions. However, none of the participants, consultants or senior registrars, knew of a body in the College that looks into its use and, if need be, produces guidelines on who should use it.

Such a powerful method may be left to people not qualified to deal with people in a vulnerable state, the case of most people seeking the help of a hypnotist. I wonder if the College can encourage colleagues who use hypnosis, and are interested in developing its use as a therapeutic tool, not as an end on its own, to get together and discuss potential uses and concerns.

A. K. DARWISH, Brynffynnon Child and Family Service, Pontypridd, Mid Glamorgan CF37 4DD 